\title{
THE GLOBAL EFFECT FOR ANTISACCADES
}

\author{
by
}

Jayalakshmi Viswanathan

B.Tech (Hons) at SASTRA University (India), 2009

\section{A THESIS SUBMITTED IN PARTIAL FULFILLMENT OF THE REQUIREMENTS FOR THE DEGREE OF}

\section{MASTER OF SCIENCE}

in

The Faculty of Graduate Studies

(Neuroscience)

THE UNIVERSITY OF BRITISH COLUMBIA

(Vancouver)

February 2012

(C)ayalakshmi Viswanathan 2012 


\begin{abstract}
$\underline{\text { Abstract }}$
In the global effect, prosaccades are deviated to a position intermediate between two targets or between a distractor and a target, which may reflect spatial averaging in the collicular map. Antisaccades differ from prosaccades in that they dissociate the locations of the stimulus and goal, and generate weaker collicular activity. We used these antisaccade properties to determine whether the global effect was generated in stimulus or goal computations, and if the global effect would be larger for antisaccades, as predicted by an origin of the effect in collicular averaging. In the first two experiments, human subjects performed antisaccades while distractors were placed in the vicinity of either the stimulus or the saccadic goal. Global effects occurred only for goalrelated and not for stimulus-related distractors, indicating that this effect emerges from interactions with motor representations. In the last experiment, subjects performed prosaccades and antisaccades with and without goal-related distractors. When the results were adjusted for differences in response latency, the global effects for rapid responses were three to four times larger for antisaccades than for prosaccades. These results were consistent with predictions of spatial averaging in a collicular model. We conclude that the antisaccade global effect shows properties compatible with spatial averaging in collicular maps, and if so, originate in layers with neural activity related to goal rather than stimulus representations.
\end{abstract}




\section{$\underline{\text { Preface }}$}

All the experiments are based on work conducted in UBC's Human Vision and Eye Movement Laboratory by Dr. Jason Barton (at the Eye Care Centre, Vancouver General Hospital), and I. I was responsible for creating the experiments, recruiting and recording subjects, data analysis, literature review and writing a preliminary draft of the work.

This work was approved by the UBC Clinical Research Ethics Board and Vancouver Coastal Health Authority. The certificate details are:

Vancouver Coastal Health Authority certificate: \#V05-0051

UBC Clinical Research Ethics Board Certificate: \# C05-0119

UBC Clinical Research Ethics Board Certificate of Renewal Approval certificate: \#H05-70119 


\section{$\underline{\text { Table of Contents }}$}

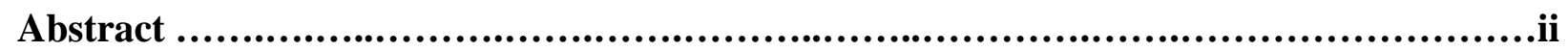

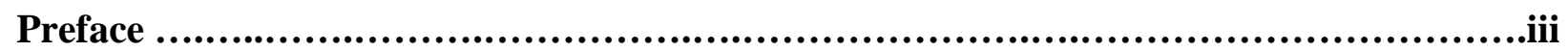

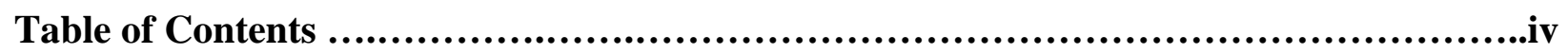

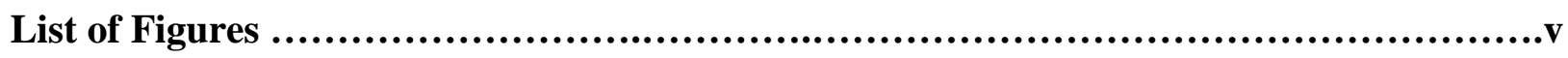

Acknowledgements ...........................................................................vi

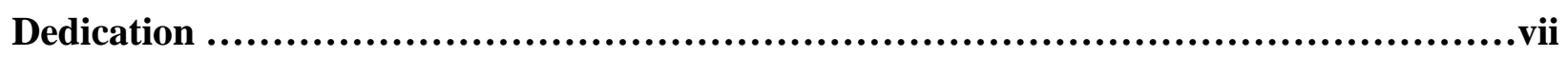

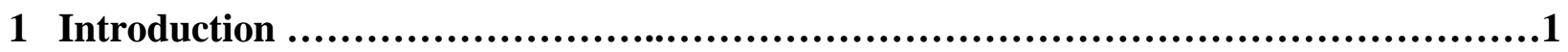

2 Experiment 1: Global effect in antisaccades for a target with stimulus vs

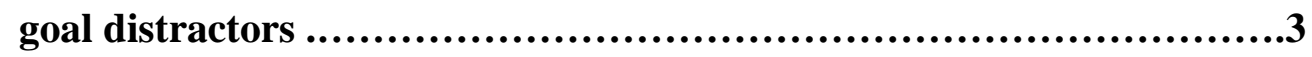

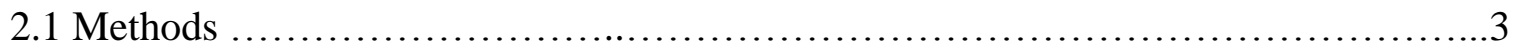

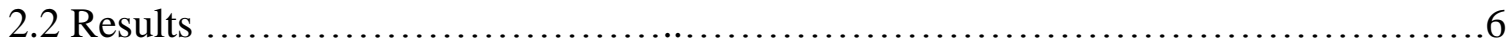

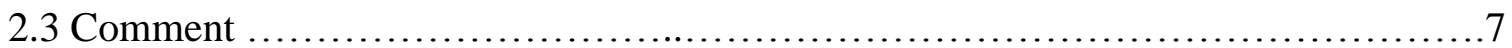

3 Experiment 2: Global effect in antisaccades for a target with variable horizontal location ..............................................8

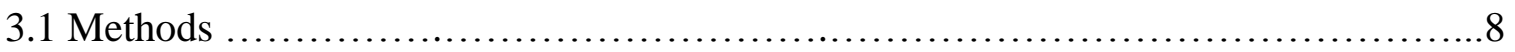

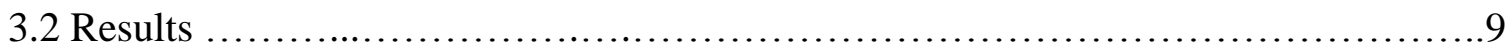

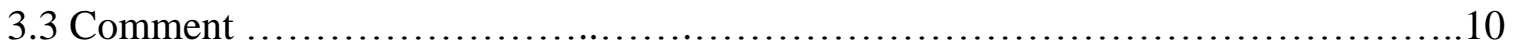

4 Experiment 3: Global effect for prosaccades vs antisaccades .............................11

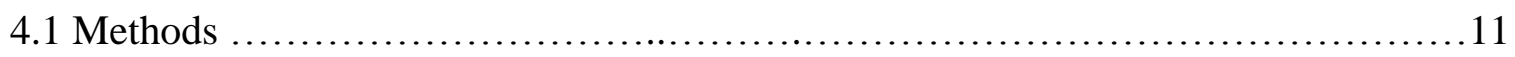

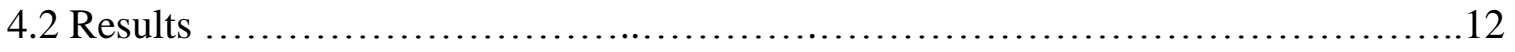

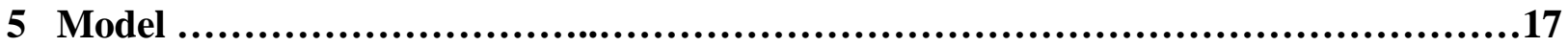

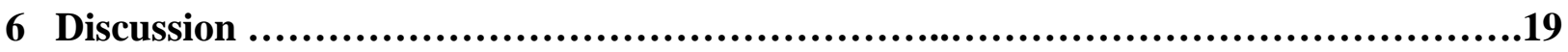

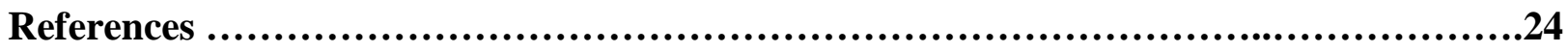

Appendix: A tectal model of averaging for the global effect ................................27 


\section{List of Figures}

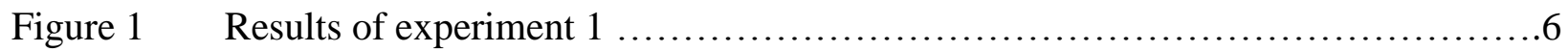

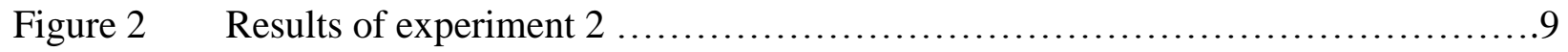

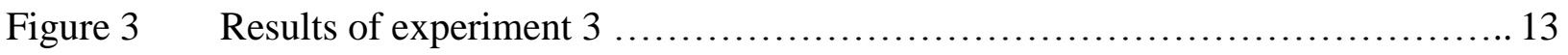

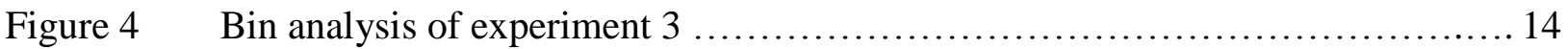

Figure 5 Bin analysis of experiment 3 in polar coordinates appropriate

to a tectal map, showing the difference between prosaccades

and antisaccades

Figure 6 Bin analysis of experiments 1 and 2 in polar coordinates appropriate to a tectal map, showing the difference between goal-related and stimulus-related distractors for antisaccades

Figure 7 Fit of tectal model predictions to the latency-binned data for short-latency prosaccades and antisaccades of experiment 3 18

Figure 8 Model of spatial averaging between a saccade on the horizontal meridian and a distractor located $20^{\circ}$ off the horizontal meridian 


\section{Acknowledgements}

I owe particular thanks to Dr. Jason JS Barton, for his encouragement and support. Research under his inspiring supervision has led me to approach hypothesis in a systematic, feasible and result oriented manner.

I thank Dr. Alan Kingstone, Dr. Todd C Handy and Dr. Todd Woodward for enlarging my vision of science and providing timely guidance and assistance.

I would like to thank CIHR for supporting me and my research through their grant CIHR MOP 81270.

I thank all the members of the Human Vision and Eye movement laboratory for the friendly, supportive and collaborative environment that has led to the growth of my ideas.

I offer my enduring gratitude to the faculty, staff and my fellow students at the UBC, who have inspired me to continue my work in this field.

Special thanks are owed to my parents, whose have supported me throughout my years of education, emotionally, morally and financially. 
To my parents 


\section{Chapter 1:}

\section{Introduction}

In the global or 'center-of-gravity' effect, a saccade directed towards two stimuli appearing simultaneously lands at an intermediate position between the two (Deubel, Wolf, \& Hauske, 1984; Findlay, 1982; F. Ottes, van Gisbergen, \& Eggermont, 1984). This occurs as long as the two are reasonable close: if they are widely separated, the amplitude of the saccade is unaffected but its latency is prolonged, the 'remote distractor effect' (Findlay, 1982; LévySchoen, 1969; Van der Stigchel, de Vries, Bethlehem, \& Theeuwes, 2011; Walker, Deubel, Schneider, \& Findlay, 1997). The global effect also occurs even if one stimulus is designated the target and the other a distractor, the two being distinguished by some property such as colour or shape (Coren \& Hoenig, 1972; Findlay, 1997; F. P. Ottes, Van Gisbergen, \& Eggermont, 1985; Walker, et al., 1997).

Electrophysiological recordings suggest that the global effect may arise from averaging of stimulus- and distractor-related activity in the tectal map (Glimcher \& Sparks, 1993), a proposal reflected in recent models based upon a population coding theory (Meeter, Van der Stigchel, \& Theeuwes, 2010). The superior colliculus is often considered an important convergent point in eye movement control, where signals from other regions such as the parietal eye fields, frontal eye fields, and substantia nigra pars reticulata $(\mathrm{SNr})$ ) are integrated to form the final saccadic signal (Hall and Moschovakis, 2004). The superior colliculus contains a topographic map in polar coordinates, and direction and amplitude of a saccade likely reflects the summed contributions of active cells within this map (A. J. van Opstal \& van Gisbergen, 1989).

Almost all research on the global effect has used prosaccades, responses directed immediately towards a suddenly appearing target. Exploring the global effect with other types of saccades may provide insights into aspects of saccadic programming. For example, global effects have also been found for memory-guided saccades (Herwig, Beisert, \& Schneider, 2010), when the subject must delay making a saccade to a briefly flashed target for several seconds. This has been interpreted as evidence that spatial averaging can occur with representations of the target location that are held in working memory.

Antisaccades are another type of saccadic response, in which gaze is directed to a point equidistant but opposite in direction to the target (Hallett, 1978); thus they involve both 
inhibition of the more natural prosaccade and programming of the novel "look-away" response (Munoz \& Everling, 2004). Two aspects of the antisaccade have particular relevance for studies of the origin of the global effect. First, the locations of the visual stimulus and the goal of the eye movement are dissociated in an antisaccade, whereas they are the one and the same for a prosaccade. This offers a direct means of determining whether the global effect arises from computations regarding stimulus location or from computations regarding the goal of the eye movement. At present this is not clear. One study has argued for a perceptual rather than motor origin because increased predictability of target location within a stimulus cluster reduced the global effect, despite the fact that the actual retinotopic position of the cluster was unpredictable (Coeffe \& O'Regan, 1987). It was suggested that this was due to prediction increasing the accuracy of spatial estimates of target position rather than advance specification of saccadic metrics. Such a conclusion, however, is only an indirect inference: the antisaccade offers a more direct method of addressing this issue.

Second, neurophysiological studies show that the target related neural activity in the Superior Colliculus is considerably weaker in antisaccades compared to prosaccades (Everling, Dorris, Klein, \& Munoz, 1999). There is a greater activity for antisaccades in the Dorsolateral prefrontal cortex (dIPFC) (Everling \& Munoz, 2000) which inhibits the buildup activity in the Superior Colliculus, further downstream in the eye movment control circuit (Sommer \& Wurtz, 2000). Studies supporting spatial averaging have examined the effects of altering the balance in salience and perceptual properties of the two stimuli (Deubel \& Hauske, 1988; Deubel, et al., 1984; Findlay, 1982; Findlay, Brogan, \& Wenban-Smith, 1993). However, the effect of altering the properties of the saccade itself has seldom been considered. Nevertheless, if the spatial averaging hypothesis is correct, altering the neural activity related to the saccade should have effects similar to altering the neural activity related to the distractor.

In our first and second experiments we asked whether a global effect is elicited by distractors in the vicinity of the saccadic goal, or by distractors in the vicinity of the stimulus. If the prior inferences are correct, that the global effect has its origin in perceptual localization, we hypothesize that a global effect will be generated by distractors near the stimulus, rather than by distractors near the saccade goal. In our third experiment we evaluated the hypothesis that antisaccades would show greater global effects than prosaccades, as predicted by spatial averaging accounts. 


\section{Chapter 2:}

\section{Experiment 1 - Global effect in antisaccades for a target with stimulus vs goal distractors}

\section{$\underline{2.1 \text { Methods }}$}

\section{Participants:}

Twelve subjects participated, 6 men and 6 women, with mean age of 26.67 years (median 26, range 21 to 35). All subjects had normal or corrected-to-normal vision and viewed all the stimuli with both eyes. None had a history of any neurological disorders. The protocol was approved by the institutional review boards of Vancouver General Hospital and the University of British Columbia, and all participants in this and the subsequent experiments gave informed consent in accordance with the declaration of Helsinki. All subjects in all experiments were naïve to the purpose of each study.

\section{Apparatus and procedure:}

Subjects sat in a room with dim lighting standardized across subjects, positioned $34 \mathrm{~cm}$ away from the computer display. Head position was maintained by a chin-rest. Eye movements were recorded by an Eyelink 1000 binocular system (SR Research Ltd, Mississauga, Canada). Stimuli and trials were programmed in SR Research Experiment Builder 1.6.1.

We used black stimuli on a white background. Stimuli were created as text objects in Arial font. The fixation cross was a ' + ' spanning $1.43^{\circ}$, font size of 30 . The target in all eye movement tasks was an ' $\mathrm{x}$ ' with height of $0.97^{\circ}$; the distractor was an ' $\mathrm{o}$ ' with a diameter of $0.97^{\circ}$. Subjects were instructed to make a saccade to a location of equal eccentricity in the opposite direction on antisaccade trials, and to ignore the distractor.

Each trial began with a fixation cross: subjects had to fixate within $2^{\circ}$ of the cross for at least $200 \mathrm{~ms}$ for the trial to progress. After an interval of $1050 \mathrm{~ms}$, the target appeared at $8^{\circ}$ eccentricity on the horizontal meridian, on either the left or right side randomly. The trial terminated when a saccade with amplitude greater than $2^{\circ}$ was detected. 
There were five distractor conditions, given in random order within each block. In the first there was no distractor. In the upper condition, a distractor appeared at $8^{\circ}$ visual angle eccentricity but displaced upwards by $20^{\circ}$ of polar angle from the horizontal meridian; in the lower condition a distractor appeared at $8^{\circ}$ visual angle eccentricity but displaced $20^{\circ}$ downwards in polar angle. In the near and far conditions distractors were located on the horizontal meridian, with the near target at $4^{\circ}$ eccentricity and the far target at $12^{\circ}$ eccentricity. There were 25 trials that appeared in 5 distractor conditions in each hemifield in each block, giving a total of 250 trials per block, and 500 trials in the entire experiment.

In the first block, all distractors appeared in the hemifield opposite to the one in which the target appeared, and hence in the vicinity of the intended goal of the antisaccade. In the second block, all distractors appeared in the same hemifield as the stimulus. Given that the global effect for prosaccades only operates over a limited spatial range, distractors in the vicinity of the antisaccade goal are likely to exert their effects on computations of desired goal position rather than target location, whereas the reverse will be true for distractors in the vicinity of the antisaccade target.

Subjects were given a break between the two blocks and, to avoid fatigue, were allowed to take breaks at other times as desired. The order of the two blocks was counterbalanced across the subjects.

\section{Analysis:}

Data was analysed by SR Research Eyelink Data Viewer 1.10.1. Saccades were detected when eye velocity reached $31 \%$, acceleration exceeded $9,100 \% \mathrm{~s}^{2}$, and position change by more than $0.15^{\circ}$. The first saccade of at least $1^{\circ}$ amplitude after target onset was considered the response. The latency was calculated as time between stimulus onset and saccade onset. Saccades with latencies less than $80 \mathrm{~ms}$ were considered anticipatory responses rather than response to the target and were excluded, as were saccades with latencies greater than $800 \mathrm{~ms}$. Saccades with a starting position outside of a $2^{\circ}$ square window around the fixation cross were discarded, as were antisaccade directional errors, defined as saccades with a horizontal vector directed towards the stimulus instead of away from it. Data was collapsed across right and left directions. 
The global effect should represent spatial averaging of the distractor with either the saccadic goal or the target. We wished to derive a simple, single summary variable in Cartesian coordinates that would characterize the global effect for each subject across all conditions with a distractor. However, this requires different signing conventions for distractors in the vicinity of the antisaccade goal than for those in the vicinity of the stimulus.

For distractors near the goal, the global effect should be a deviation of saccadic endpoint towards the distractor, compared to the endpoint when there is no distractor. Hence for the near distractor, a reduction in the horizontal projection of the saccade vector would be the predicted global effect, while for a far distractor it would be an increase in that projection. Similarly, for an upper distractor, the global effect would cause mainly an upward deviation of the vertical projection of the saccade vector, whereas for a lower distractor it would be a downward deviation. Thus for each of the distractor conditions, we calculated the global effect as a difference between the distractor and no-distractor condition as follows. For the near-distractor condition we subtract the mean horizontal component of its saccades from those of saccades in the no-distractor condition, whereas for the far-distractor condition, we do the reverse. For the lower-distractor condition we subtract the mean vertical component of its saccades from those of saccades in the no-distractor condition, whereas for the upper-distractor condition, we do the reverse. Thus a positive value always represents the predicted global effect of deviation towards a distractor

For distractors near the stimulus, far and near distractors around the stimulus should lead to similar effects as the far and near distractors located near the antisaccade goal. That is, a near distractor should cause perceptual localization of the stimulus to be underestimated, leading to a smaller antisaccade, just as a near distractor in the goal condition would do. However, the vertical distractors should lead to effects opposite to those seen with distractors around the antisaccade goal. Spatial averaging of the stimulus with an upper distractor would lead to misperception of the stimulus as being above the horizontal meridian, with the resulting antisaccade being directed below the horizontal meridian in the contralateral hemifield. (In contrast, a distractor below the antisaccade goal would cause upward displacement of the estimated goal location.) Hence, for distractors near the stimulus, we calculated global effects for the four different distractor conditions in a similar manner as with the goal condition, but reversed the sign for the upper and lower distractor conditions. 
As our goal was not to investigate any differential effects of distractor direction, we averaged the global effects across all four distractor conditions to give a single estimate of the global effect for each subject in each of the two conditions. We subjected the group data to a ttest for the presence of a significant global effect, as well as a paired t-test to determine if the global effect under goal and stimulus conditions differed.

\section{$\underline{2.2 \text { Results }}$}

There was a significant global effect for the goal condition $\left(1.26^{\circ}\right.$, s.d. $1.17, \mathrm{t}(11)=$ $3.75, \mathrm{p}<0.0016$, Figure 1). In contrast, there was no global effect for the stimulus condition ($0.004^{\circ}$, s.d. $\left.0.25, \mathrm{t}(11)=0.065, \mathrm{p}=0.47\right)$. There was a significant difference between the global effects in the stimulus and goal conditions $(\mathrm{t}(11)=3.51, \mathrm{p}<0.005)$.
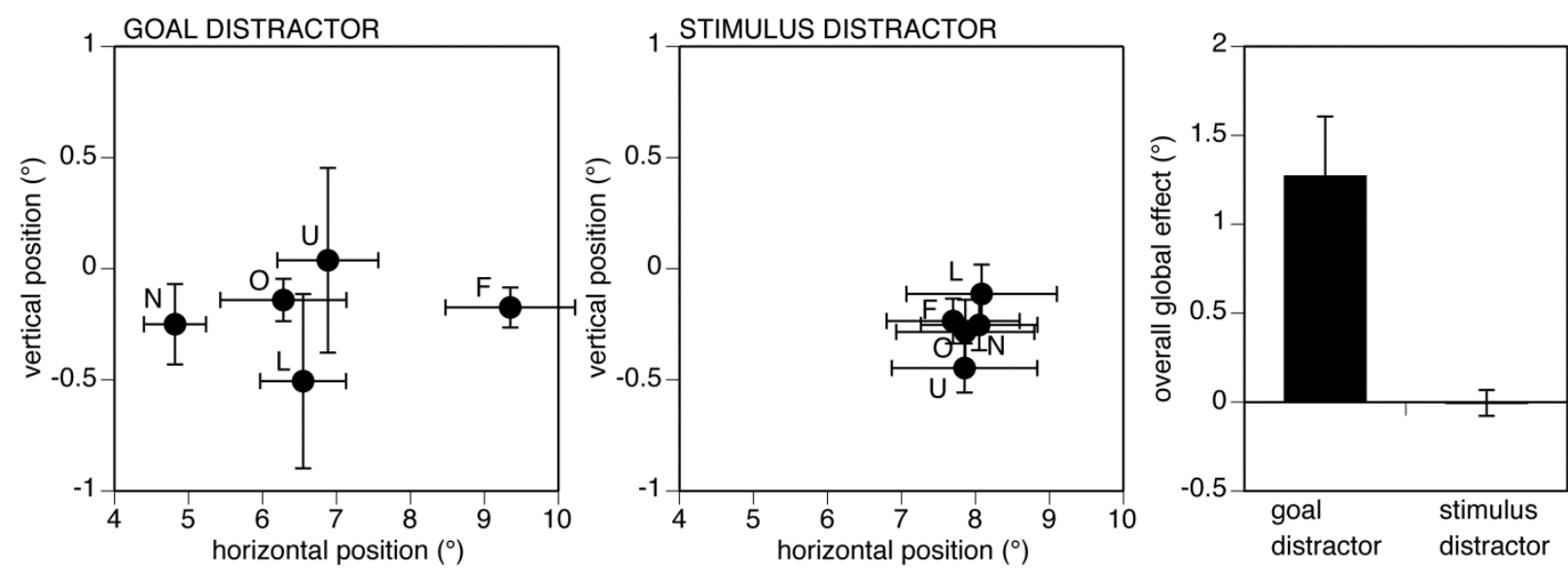

Figure 1. Results of experiment 1. A) Cartesian plot of results of the block with distractors surrounding the desired antisaccade goal. Mean saccade endpoints in the five distractor conditions $(\mathrm{U}=$ upper, $\mathrm{L}=$ lower, $\mathrm{N}=$ near, $\mathrm{F}=$ far, $0=$ none). Right and left-sided trials have been collapsed and portrayed in the right hemifield. Error bars are one standard error of x and $\mathrm{y}$ Cartesian coordinates. B) Plot of results of the block with distractors surrounding the antisaccade target stimulus. C) Summary global effect variable collapsing across the four conditions with distractors. Error bar shows one standard error. 


\subsection{Comment}

These results show that there is a significant global effect for distractors located in the vicinity of the goal of the antisaccade task, but none for distractors in the vicinity of the antisaccade stimulus. This suggests strongly that the global effect induced by distractors is due to their modulation of computations of the desired eye movement, rather than computations of stimulus location. However, before concluding that there is no effect of distractors on stimulus processing, we wished to consider the possibility that this result stemmed from the fact that predictable factors in our task design might have minimized the computations of stimulus location required of our subjects. While the side of target appearance was random, its eccentricity was always $8^{\circ}$. This uniformity might have meant that subjects did not compute the location of the stimulus on each trial. Rather, target appearance may have been used merely to indicate the direction of the desired movement (right versus left) and as an onset signal to simply trigger a saccade whose amplitude they had already pre-programmed. To exclude this possibility, we created a second experiment. 


\section{Chapter 3:}

\section{Experiment 2 - Global effect in antisaccades for a target with variable horizontal location}

In this study, we varied the horizontal position of the target randomly from trial to trial, so that subjects were forced to compute the eccentricity of the stimulus to execute correctly the command to make an antisaccade of equal eccentricity in the direction opposite to the target.

\section{$\underline{3.1 \text { Methods }}$}

Participants were 12 subjects, 5 men and 7 women with mean age of 29.17 years (median 28.5 , range 22 to 39 ).

We used the same apparati as in Experiment 1. Stimuli for fixation crosses, targets and distractors were identical to Experiment 1. On any given trial, the target could appear at one of five eccentricities, $6,7,8,9$ or $10^{\circ}$, left or right, with eccentricity and direction determined randomly between trials. We used only 3 distractor conditions: near, far and none. In the near distractor condition, a distractor appeared $4^{\circ}$ nearer to fixation than the target or goal, while in the far distractor condition, it appeared $4^{\circ}$ more eccentric that the target or goal. To limit the number of trials to a manageable number, we omitted the upper and lower distractor conditions. As in experiment 1 , there were two blocks, whose order was counterbalanced across subjects. In one, the distractors were located in the vicinity of the goal of the antisaccade, while in the second the distractors surrounded the vicinity of the target stimulus. Each of the three distractor conditions for each of the five target eccentricities appeared 16 times in each block, 8 in each hemifield, giving 240 trials per block, for a total of 480 trials in the whole experiment.

Analytic methods followed those of experiment 1 . We collapsed the results across the different target eccentricities and the right and left sides. (For display purposes only, we plotted the results as if the target location had been $8^{\circ}$ for all trials.) 


\section{$\underline{3.2 \text { Results }}$}

There was a significant global effect for the goal condition $\left(1.40^{\circ}\right.$, s.d. $0.86, \mathrm{t}(11)=5.62$, $\mathrm{p}<0.0002$ ), whose magnitude is comparable to that seen in Experiment 1, even though this second experiment lacked upper and lower distractor conditions (Figure 2). In contrast, there was still no global effect for the stimulus condition $\left(-0.091^{\circ}\right.$, s.d. $\left.0.37, t(11)=0.86, p=0.41\right)$. There was a significant difference between the global effects in the stimulus and goal conditions $(t(11)$ $=3.51, \mathrm{p}<0.005)$.
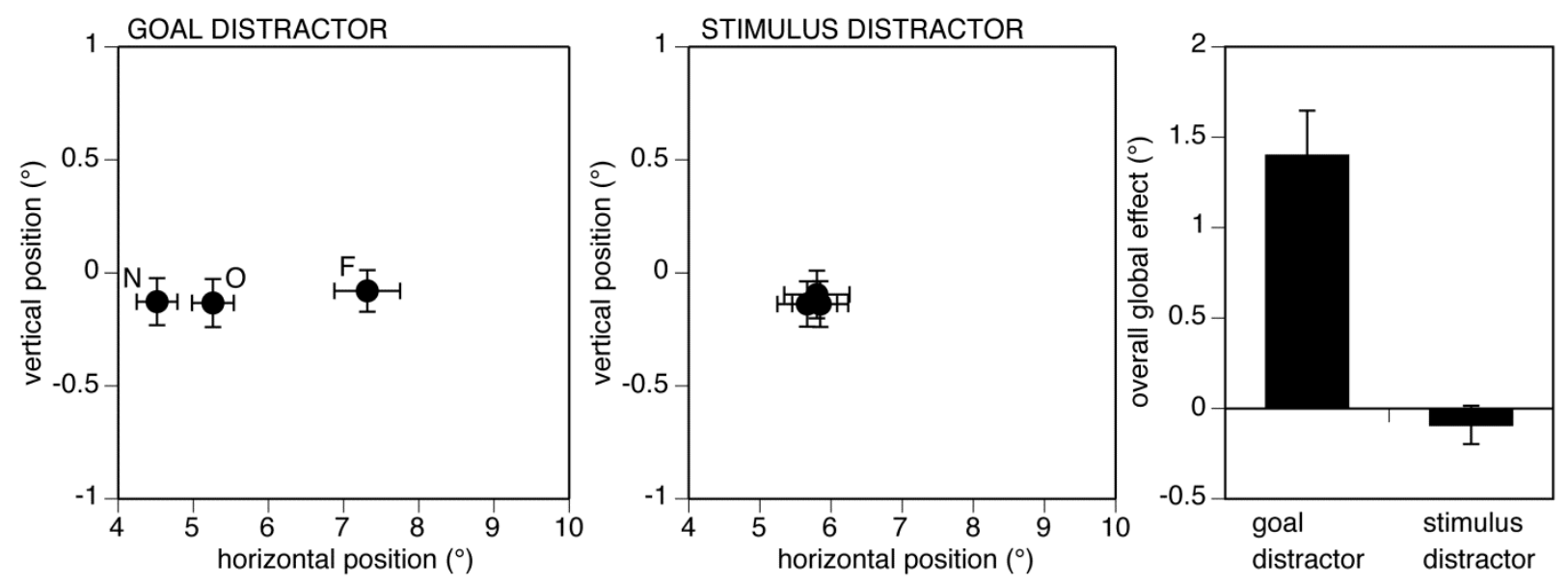

Figure 2. Results of experiment 2. A) Cartesian plot of results for blocks in which the distractors are located near the goal of the antisaccade, where $\mathrm{N}=$ near distractor condition, $\mathrm{F}=$ far distractor condition, and $0=$ no distractor condition. Error bars indicate one standard error. B) Plot of results for the blocks in which the distractors are located near the target stimulus. C) Summary global effect variable collapsing across the two conditions with distractors. Error bar shows one standard error. 


\section{$\underline{3.3 \text { Comment }}$}

This experiment shows that, even when subjects are forced by stimulus uncertainty to compute target eccentricity to generate an antisaccade of the appropriate amplitude, distractors located in the vicinity of the target still fail to generate any global effect. On the other hand, we replicate the global effect seen in Experiment 1 when the distractors surrounded the goal location. Thus these results indicate that the global effect for saccades arises mainly if not solely in the computation of desired saccadic endpoint, rather than the estimate of stimulus location. 


\section{Chapter 4:}

\section{Experiment 3 - Global effect for prosaccades vs antisaccades}

Our first two experiments confirmed that antisaccades can show a global effect with goalrelated distractors. Hence this is consistent with spatial averaging between the activity generated at the location of the distractor and that generated by the antisaccade motor plan. This opens the possibility of exploring the impact of variations in saccadic motor programming on the global effect. To date, most studies of spatial averaging have manipulated the perceptual properties of the two simultaneously appearing stimuli (Deubel \& Hauske, 1988; Deubel, et al., 1984; Findlay, 1982; Findlay, et al., 1993). The antisaccade paradigm offers an opportunity to assess how it varies with differences in goal-related activity. Primate studies of the superior colliculus have shown that the saccade-related peak of neural activity for an antisaccade is about a third less than that for a prosaccade (Everling, et al., 1999). If the superior colliculus is the site of the spatial averaging responsible for the global effect, then one prediction is that the global effect should be larger for an antisaccade than for a prosaccade. In this last experiment, we measured the global effect for prosaccades and antisaccades in the same subjects, and compared the results to predictions of a current model of collicular neural activity.

\subsection{Methods}

Participants were 12 subjects, 4 men and 8 women, with mean age of 24.75 years (median 23.5, range 21 to 38 ).

We used the same apparati as in Experiments 1 and 2. Stimuli for fixation crosses, targets and distractors were identical to those in Experiments 1 and 2. The target appeared at $8^{\circ}$ eccentricity on the horizontal meridian, to either the left or right randomly. We used the same five distractor conditions and locations as in experiment 1: upper, lower, near, far and none. There were 50 trials for each of the 5 distractor conditions, 25 on the right and 25 on the left, for a total of 250 trials per block. There were two blocks, given in counterbalanced order across subjects. In the prosaccade block, subjects looked to the target as soon as it appeared. In the 
antisaccade block, subjects were instructed to make a saccade of equal amplitude in the direction opposite to the target. Distractors appeared in the vicinity of the goal location.

As in experiment 1 , the analysis collapsed the results across right and left sides and averaged the effects across all four distractor conditions to give a single estimate of the global effect for each subject in each of the two conditions. We subjected the group data to a t-test for the presence of a significant global effect, as well as a paired t-test to determine if the global effect under goal and stimulus conditions differed.

However, a possible confound in a comparison between prosaccades and antisaccades is that these responses differ in latency, with antisaccades having longer latencies than prosaccades (Hallett \& Adams, 1980). It is known that the global effect varies with prosaccade latency, being maximal for rapid responses (Eggert, Sailer, Ditterich, \& Straube, 2002; F. P. Ottes, et al., 1985). To correct for latency variations, we performed a bin analysis on the whole-group data, grouping the saccades into latency bins of 40ms width, beginning with a bin between 160 and $200 \mathrm{~ms}$, and ending at $400 \mathrm{~ms}$, with all of the few saccades with latencies longer than $400 \mathrm{~ms}$ grouped into the same terminal bin.

\section{$\underline{4.2 \text { Results }}$}

There was a significant global effect for both prosaccades $\left(1.05^{\circ}\right.$, s.d. $0.28, \mathrm{t}_{(11)}=13.31$, $\mathrm{p}<<0.0001)$ and antisaccades $\left(0.96^{\circ}\right.$, s.d. $\left.0.70, \mathrm{t}_{(11)}=4.74, \mathrm{p}<0.0004\right)$. There was no significant difference between the global effects in the stimulus and goal conditions $(\mathrm{t}(11)=0.56, \mathrm{p}=0.59)$. However, the variance for the antisaccade global effect was greater than that for prosaccades $\left(\mathrm{F}_{(11,11)}=6.43, \mathrm{p}<.005\right)$, which likely is a simple reflection of the fact that antisaccade accuracy is more variable than prosaccade accuracy, as can be seen for both distractor and no-distractor conditions in Figure 3. 

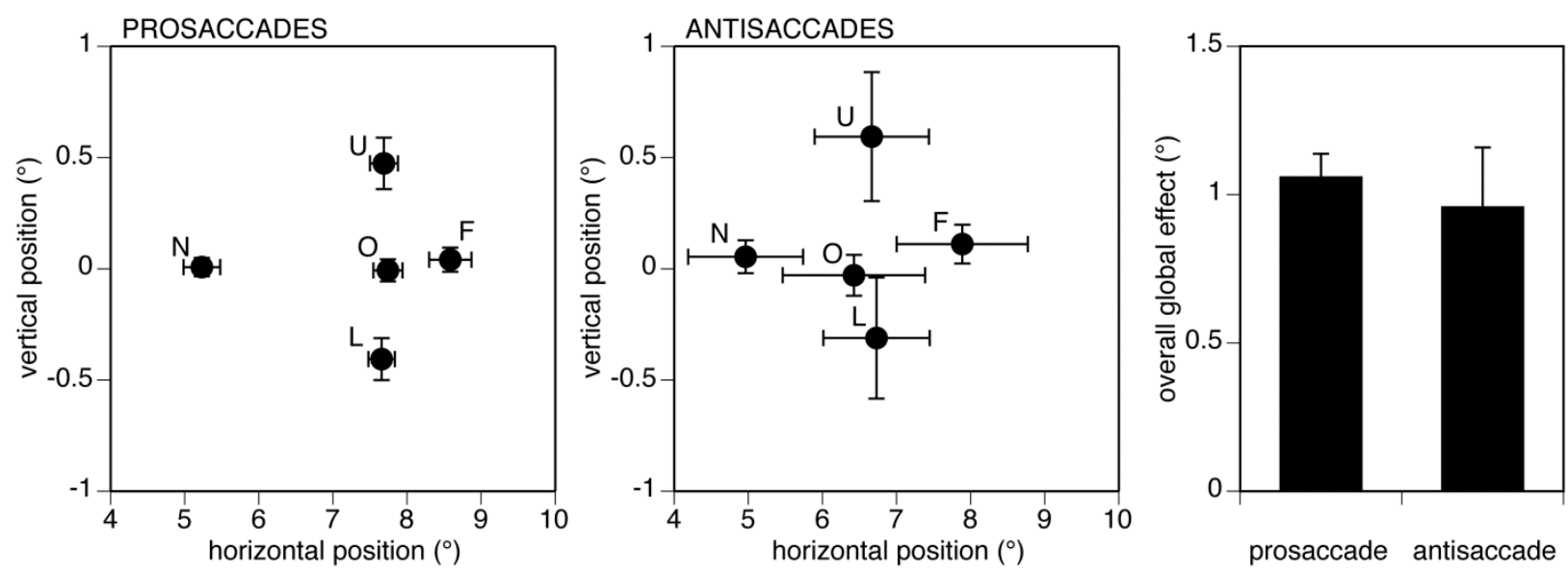

Figure 3. Results of experiment 3. A) Cartesian plot of results for the prosaccade blocks. Mean saccade endpoints in the five distractor conditions $(\mathrm{U}=$ upper, $\mathrm{L}=$ lower, $\mathrm{N}=$ near, $\mathrm{F}=$ far, 0 = none. Error bars indicate one standard error. B) Plot of results for the antisaccade blocks, in which the distractors are located near the antisaccade goal. C) Summary global effect variable collapsing across the five conditions with distractors. Error bar shows one standard error.

This first analysis might appear to disprove the prediction of a greater global effect for antisaccades than prosaccades. However, it fails to take into account differences in the latency distribution between antisaccades and prosaccades, which gains importance by the fact that the global effect is mainly seen for rapid responses. When we examined the data by latency bins, three findings emerged (Figure 4). First, we replicate the previous observations (Eggert, et al., 2002; F. P. Ottes, et al., 1985) that the global effect declines in magnitude with increasing saccadic latency. This has been explained as a consequence of the increased time available for target-distractor discrimination in long latency saccades leading to increased accuracy. Second, for any given latency bin, the global effect is on average about 1.6 times larger for antisaccades than for prosaccades (mean difference over bins $=0.61^{\circ}$, s.d. $0.41, \mathrm{t}_{(6)}=3.62, \mathrm{p}<.009$ ). Third, the reason why similar global effects are found for prosaccades and antisaccades in the first analysis, which did not take latency into account, becomes clear when we consider the numbers of prosaccades and antisaccades in each latency bin. Most prosaccades had latencies between 160 and 240ms, when the prosaccade global effect was greatest, while the larger proportion of antisaccades had latencies above 280ms, when the antisaccade global effect had declined. 

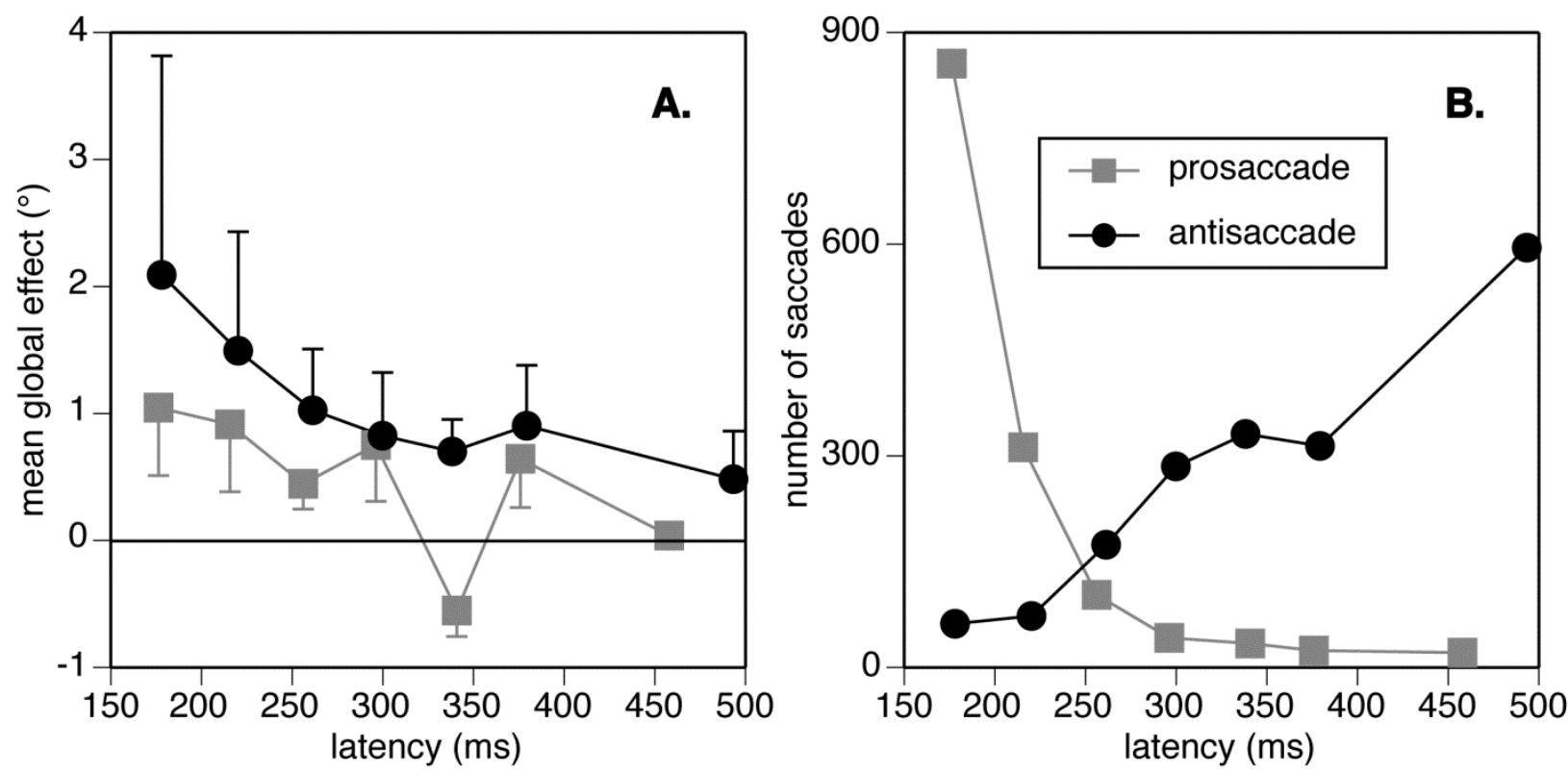

Figure 4. Bin analysis of Experiment 3. A) Mean global effect averaged across all four distractor-present conditions and across all subjects, with results divided into 40ms-wide bins, plotted against the mean latency for saccades in those bins. Error bars indicate one standard error. B) Number of saccades made by all subjects and summed over all five conditions in each latency bin, showing that most prosaccades have latencies less than $240 \mathrm{~ms}$, while most antisaccades have latencies more than $280 \mathrm{~ms}$.

The difference between prosaccades and antisaccades becomes even clearer when we illustrate the data in polar coordinates, as appropriate for a tectal map (Figure 5). The upper and lower distractors located at $20^{\circ}$ polar angle would be equivalent in distance away from the horizontal meridian in this map, and so we collapse across their data. However, the near and far distractors differ in the non-linear coordinates of tectal distance, and so for these we illustrate only the data for the near condition. These show an even more marked difference between the global effect for antisaccades and prosaccades for short latency responses, with the antisaccade global effect being 3 to 4 times larger than that for prosaccades. 

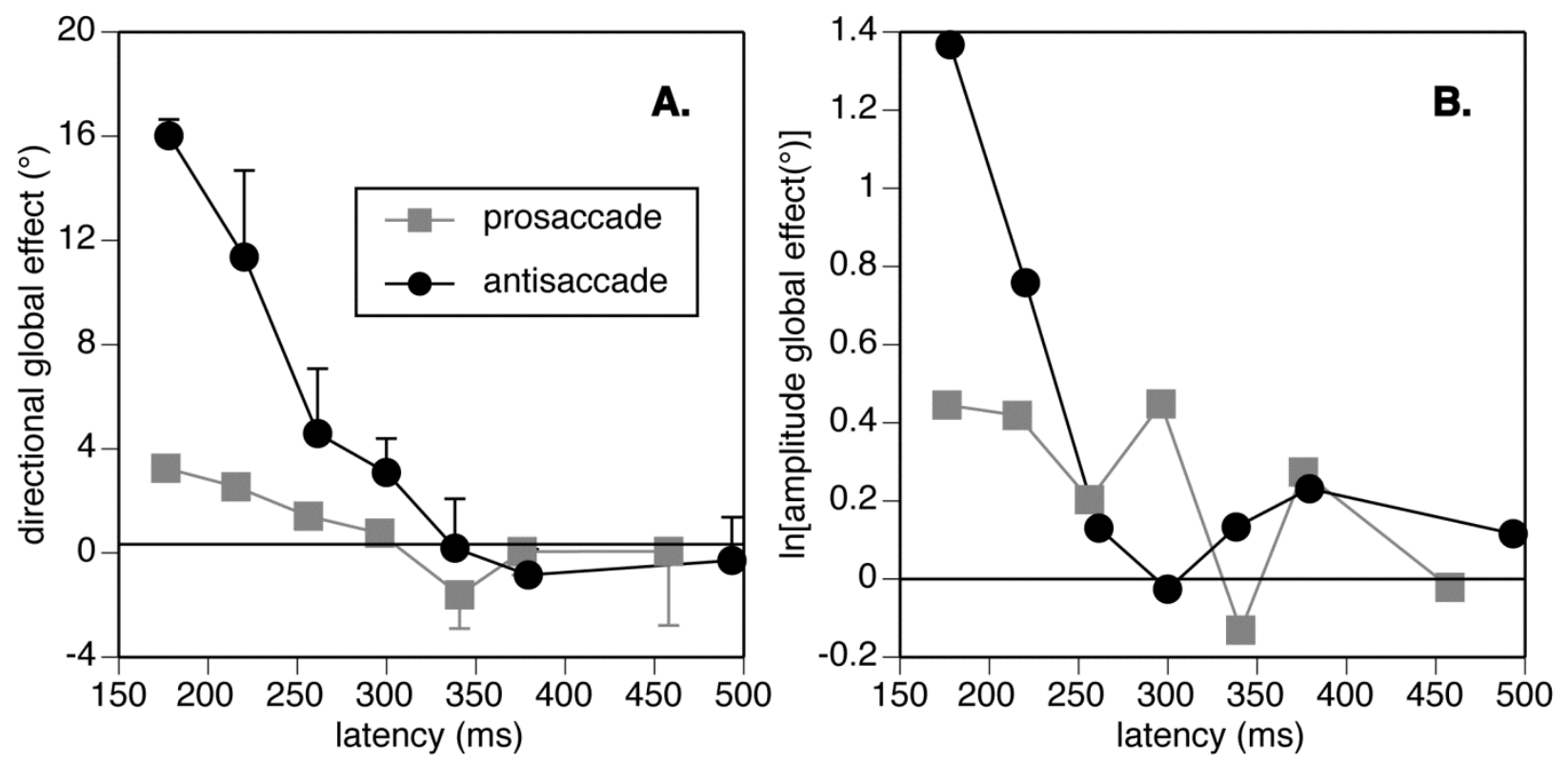

Figure 5. Bin analysis of experiment 3 in polar coordinates appropriate to a tectal map, showing the difference between prosaccades and antisaccades. A) Mean global effect averaged for both upper and lower distractors and expressed in degrees of polar angle, averaged across all subjects, with results divided into 40ms-wide bins, plotted against the mean latency for each bin. Error bars indicate one standard error. B) Mean global effect for the near distractor in visual angle and depicted logarithmically. In both graphs, there is a stronger global effect for antisaccades at short latencies.

Because this polar-expressed bin analysis was particularly effective at revealing a significant antisaccade global effect at short-latencies, we re-visited the data for experiments 1 and 2 to ensure that we had not missed a global effect from stimulus-related distractors in shortlatency antisaccades, by our strategy of averaging responses over all response latencies. While a bin analysis on these data replicated the findings of large global effects for short-latency antisaccades with goal-related distractors, we still did not see any evidence of a global effect with stimulus-related distractors (Figure 6). 

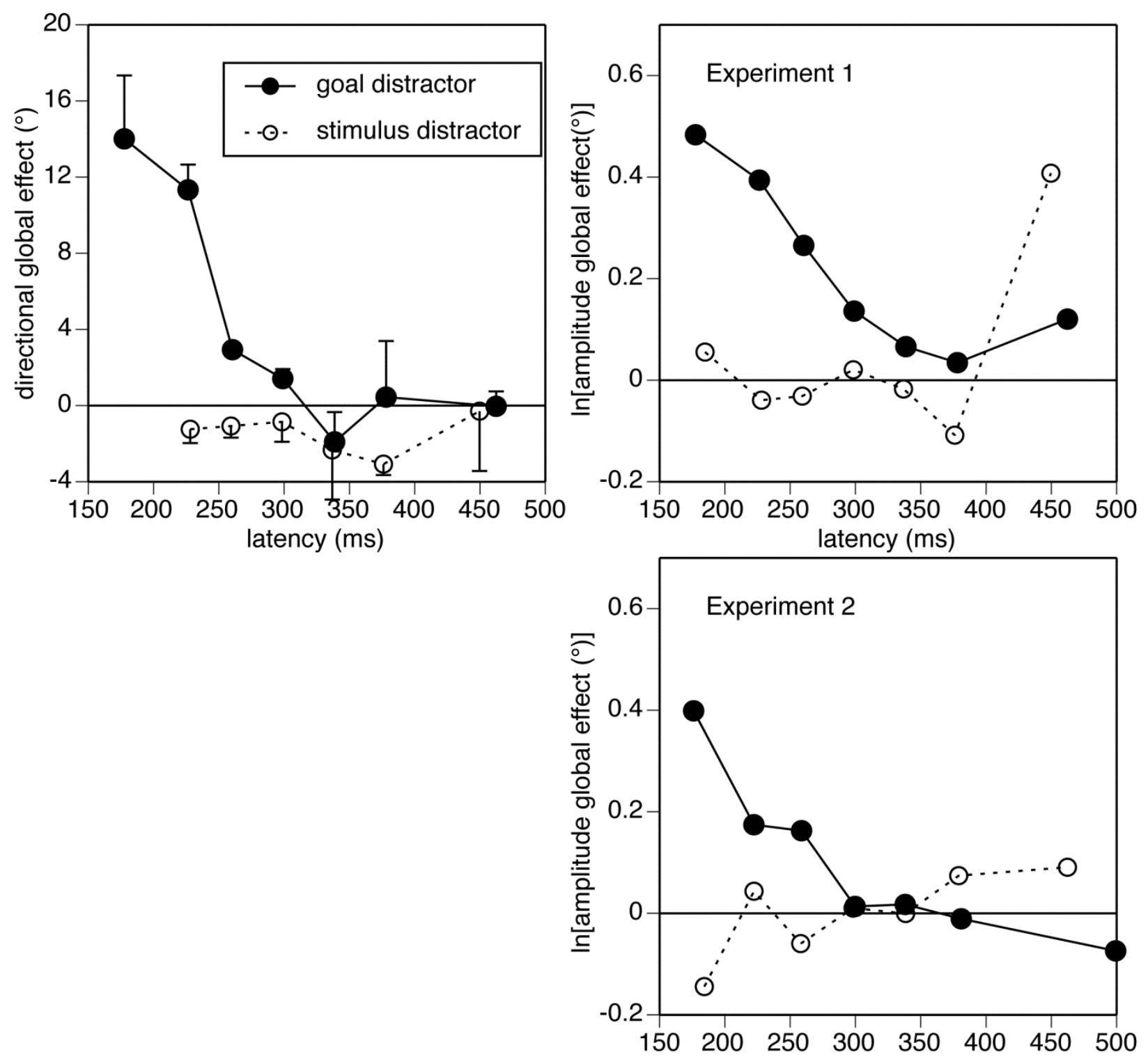

Figure 6. Bin analysis of experiments 1 and 2 in polar coordinates appropriate to a tectal map, showing the difference between goal-related and stimulus-related distractors for antisaccades. A) Experiment 1, mean global effect averaged for both upper and lower distractor and expressed in degrees of polar angle, averaged across all subjects, with results divided into 40ms-wide bins, plotted against the mean latency for each bin. Error bars indicate one standard error. B) Experiment 1, mean global effect for the near distractor in visual angle and depicted logarithmically. C) Similar data for the near distractor in Experiment 2. It is evident that there is a strong global effect for goal-related but not stimulus-related distractors at short latencies. 


\section{Chapter 5:}

\section{Model}

The data of experiment 3 are consistent with the qualitative hypothesis that antisaccade should have greater global effect for antisaccades than prosaccades. We also examined their quantitative fit with predictions from models of neural activity in the superior colliculus (Appendix). As for our polar data, for simplicity we limited ourselves to examining the global effects for directional angle. We computed the center-of-mass of a function that spatially averaged in a model collicular map the neural activity generated by both a prosaccade and a nearby distractor of equal eccentricity but $20^{\circ}$ away in directional angle. We then examined how different values of distractor-related activity relative to prosaccade activity shifted the center of mass. We identified values that created center-of-mass shifts that approximated the magnitude of global effects for directional angle seen in the prosaccade data in the different latency bins: thus, at latencies of $160 \mathrm{~ms}$, distractor-related activity in the motor map is equivalent to about $40 \%$ of prosaccade-related activity, but that this decays rapidly to about $15-17 \%$ of prosaccade activity by $240 \mathrm{~ms}$. We then examined what these predicted for global effects for antisaccades in the same latency bins. The results show a fairly good fit to our empiric data for directional global effects, with some underestimation of the effects found in the earliest latency bin (Figure 7). 


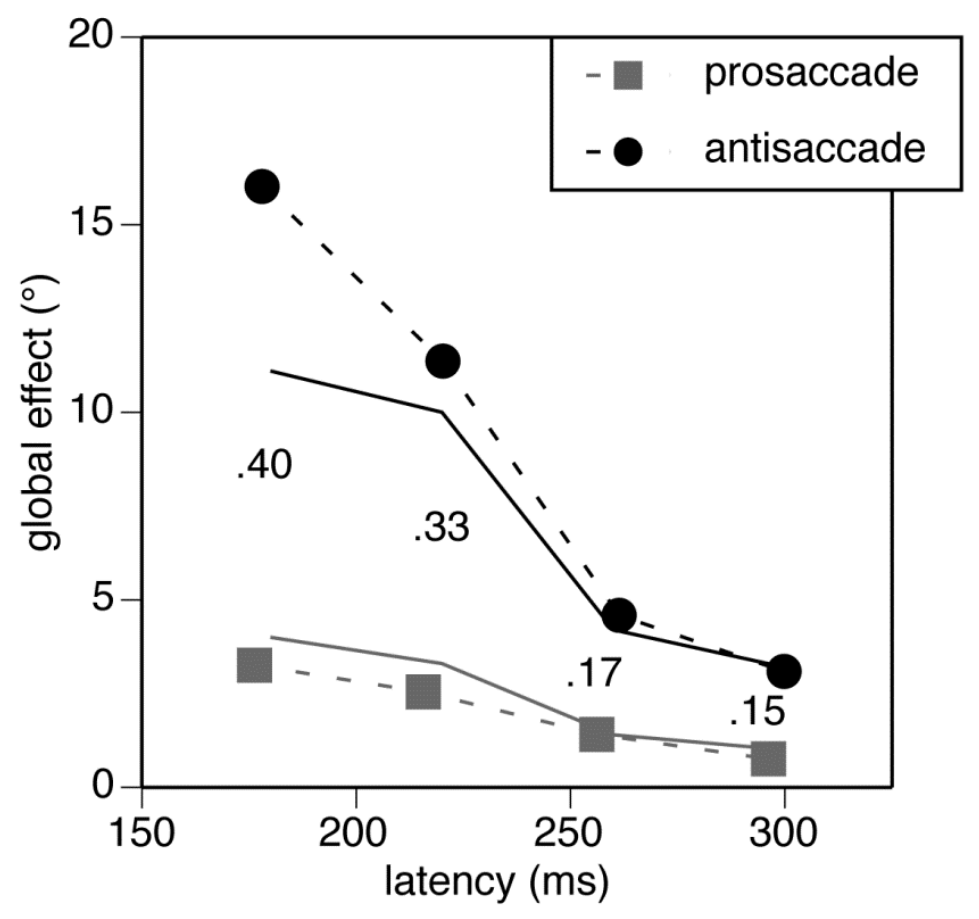

Figure 7. Fit of tectal model predictions (see Appendix) to the latency-binned data for short-latency prosaccades and antisaccades of experiment 3. Data shown for upper and lower distractors that leads to a global effect that causes a deviation in polar angle. Solid lines indicate model predictions, while symbols connected by dashed lines indicate the experimental data. Numbers indicate the weight of distractor-related activity relative to prosaccade-related activity. 


\section{Chapter 6:}

\section{Discussion}

Our experiments showed first that, when stimulus and goal activity are dissociated by asking subjects to perform an antisaccade, a global effect is generated by distractors in the vicinity of the goal, and not in the vicinity of the stimulus. We conclude that the global effect reflects a spatial averaging process that involves representations of motor goal programming, rather than stimulus localization. Second, when the temporal dynamics of the global effect are taken into account, our findings confirm the prediction from spatial averaging hypotheses that antisaccades should have a larger global effect than prosaccades. We evaluated our data with current models of the superior colliculus and found good quantitative fits with spatial averaging of neural activity in the tectal map. While our behavioural data cannot prove that the spatial averaging responsible for the global effect is occurring in the superior colliculus, they are consistent with this hypothesis, and point to goal-related neural activity as the most likely candidate for such averaging within this structure.

Early studies identified at least two effects generated by distractors or simultaneous stimuli during a saccade task (Walker, et al., 1997). Distractors distant from the stimulus increased the latency of saccades but did not affect their spatial metrics, an effect that was attributed to inhibitory collicular projections. Distractors near to the stimulus cause a global effect without affecting latency. This was considered more likely due to the spatial tuning of stimulus responses and motor discharges in the superior colliculus, which have an extent of about $30^{\circ}$ : hence two events less than $30^{\circ}$ apart will generate overlapping patterns of neural activity, leading to spatial averaging (Walker, et al., 1997). This combination of long-range inhibition and short-range averaging persists in current competitive integration models of saccade generation (Trappenberg, et al., 2001(Godijn \& Theeuwes, 2002).

If spatial averaging for the global effect occurs in the superior colliculus, a further question is where and what type of tectal activity is involved. Anatomically, the superior colliculus has 3 functionally distinct layers - the superficial, intermediate and deep layers (Mohler \& Wurtz, 1976; Sparks \& Hartwich-Young, 1989; Wurtz \& Mohler, 1976). Neurons can have visual, quasi-visual, motor activity or a combination of these (Mays \& Sparks, 1980), and 
can have burst or build-up temporal patterns (D. Munoz \& R. Wurtz, 1995). Burst and build-up neurons with motor activity or motor and visual activity predominate in the intermediate layer (D. Munoz \& R. Wurtz, 1995; D. P. Munoz \& R. H. Wurtz, 1995b), whereas the superficial layer has mainly neurons responsive to the visual stimulus (Mays \& Sparks, 1980). By showing that the global effect is specifically linked to programming of the motor goal and not to the visual stimulus, our results implicate neurons with motor activity as the likely site of the spatial averaging that culminates in the global effect.

This is consistent with a study of distractor effects in the intermediate layers of the superior colliculus (Dorris, Olivier, \& Munoz, 2007). This study used a highly predictable saccadic paradigm to generate strong motor build-up preparatory activity before stimulus onset, and presented distractors $100 \mathrm{~ms}$ before the target stimulus so that distractor effects could be observed before any visual activity was generated by the target stimulus. It found that distractors interacted with the build-up of goal-related activity before the onset of stimulus-related activity, and was correlated with the frequency of erroneous saccades towards the distractor. While this is not quite equivalent to the global effect, it does show that distractor-induced visual activity interacts with saccadic motor preparation. This interaction with buildup activity is also of note because in antisaccade paradigms, buildup activity is linked to motor preparation and not with stimulus processing (Everling, et al., 1999), and buildup activity directly influences the activity of pre-motor neurons in the brainstem (D. Munoz \& R. Wurtz, 1995).

Prior observations have often assumed that the global effect may arise in perceptual computations of stimulus location. Indeed, many studies have reported its modulation by the balance of perceptual salience between simultaneous stimuli as evidence of spatial averaging (Deubel \& Hauske, 1988; Deubel, et al., 1984; Findlay, 1982; Findlay, et al., 1993). However, it is likely that for prosaccades, alterations in the strength of neural activity generated by the stimulus will also be reflected in changes in goal-related activity (Everling, et al., 1999; D. P. Munoz \& R. H. Wurtz, 1995a). Hence these reports cannot exclude the possibility that spatial averaging is occurring in goal-related rather than stimulus-related computations. Another study found that making target location predictable within a stimulus cluster reduced the global effect, even when advance programming of a specific saccadic vector was precluded by making the retinotopic location of the cluster variable (Coeffe \& O'Regan, 1987). While this suggests that the modulation had a perceptual origin, this does not prove that the global effect is itself 
perceptual in nature. On the other hand, secondary corrective saccades and non-ocular motor perceptual localization tasks to flashed stimuli do not show the global effects seen in primary saccades (Eggert, et al., 2002), suggesting that "the primary saccade is based on a specific target acquisition process that differs from that used for spatial perception and for the programming of memory-guided corrective saccades" (p. 2969). Our results would suggest that the global effect for the primary saccade reflects averaging in programming of the saccadic goal, and not in stimulus processing, which explains why perceptual localization is unaffected by a distractor. Given that secondary saccades occur later than primary saccades, their lack of a global effect is simply consistent with the ephemeral nature of distractor-related visual activity, which reduces their influence on saccades with longer latencies (Findlay, 1982; F. P. Ottes, et al., 1985).

If the global effect reflects spatial averaging in goal-related activity in the superior colliculus, a straightforward prediction is that its magnitude will be increased by manipulations that reduce neural activity at the saccadic goal. Antisaccades generate activity in collicular buildup and burst neurons that is only a third of that generated by prosaccades (Everling, et al., 1999). After adjusting for saccadic latency, our results contrasting prosaccades and antisaccades were consistent with this prediction, and proved to be a reasonable quantitative fit to estimates derived from a population-coding model of saccadic generation in the superior colliculus (Trappenberg, Dorris, Munoz, \& Klein, 2001; A. J. van Opstal \& van Gisbergen, 1989). While our model parameters where chosen based on the best available monkey data, other parameter combinations may provide an even better fit between the model and the human data, given that the metrics of the human superior colliculus likely differ from those in the monkey (F. P. Ottes, Van Gisbergen, \& Eggermont, 1986).

A parallel observation to our results involves the study of distractor-induced deviations in saccadic trajectory. When a distractor is located near the middle of the path of a saccade rather than its endpoint, it causes the flight of the saccade to deviate away from it (Doyle \& Walker, 2001; Godijn \& Theeuwes, 2004; McSorley, Haggard, \& Walker, 2005; Van der Stigchel \& Theeuwes, 2005). This too has been modeled as an integration of distractor and goal-related activity in the superior colliculus, affecting primarily the direction of saccadic initiation (Meeter, et al., 2010). Again, we predicted that this should result in significantly greater trajectory deviation for saccades with reduced goal-related activity. This indeed was found for both antisaccades and memory-guided saccades, with up to four times as much deviation for these 
responses as for prosaccades (van Zoest, Van der Stigchel, \& Barton, 2008). Thus, our results for the global effect with antisaccades are consistent with this work on trajectory deviation by distractors. Whether global effects are similarly larger for memory-guided saccades than prosaccades is not yet known: the recent demonstration of a global effect for memory-guided saccades did not include a prosaccade condition for comparison (Herwig, et al., 2010).

Although our results are consistent with the prevailing view that the global effect reflects spatial averaging in a tectal map, they cannot prove this view, nor do they exclude potential contributions or modulation from other cerebral regions. Cortical effects are suggested by the observation of paradoxical blindsight global effects in hemianopia, with deviation away from distractors located in the blind field (Van der Stigchel, van Zoest, Theeuwes, \& Barton, 2008), as well as findings of reduced global effects in the contralateral hemifield and increased global effects in the ipsilateral hemifield in a patient with hemineglect (Walker \& Findlay, 1996). Other evidence for top-down attentional effects include the finding that the spatial extent of the global effect for prosaccades is more limited than the extent of the global effect for 'capture saccades' (saccades to irrelevant distractors), suggesting that the former is modulated by top-down attentional control (Van der Stigchel, et al., 2011). Others have shown that the global effect is modified by prior probability (He \& Kowler, 1989). While extra-collicular mechanisms are not included in most current collicular models (A. J. van Opstal \& van Gisbergen, 1989), inclusion of such elements may improve their predictions regarding human behaviour.

Our prosaccade and antisaccade data also yielded some data of interest on the quantitative dynamics of distractor-related activity (Figure 7). Previous studies have long observed that the global effect is greater for saccades with short latencies (Findlay, 1982; Findlay \& Gilchrist, 1997; F. P. Ottes, et al., 1985), less for saccades with latencies greater than 200ms (Eggert, et al., 2002), or when a response delay of 350ms is imposed (Coeffe \& O'Regan, 1987). Our model estimations of the strength of distractor activity relative to concurrent prosaccade activity provide more detail on this dynamic, with distractor-related activity in the motor map equivalent to about $40 \%$ of prosaccade-related activity at $160 \mathrm{~ms}$ latencies, but rapidly declining to negligible levels at latencies of about $350 \mathrm{~ms}$. As yet, and has noted by others, there is little data on the temporal dynamics of visual ('exogenous') activity in the superior colliculus (Trappenberg, et al., 2001): however, one recent study suggested that the abrupt-onset visual signals from a distractor generate a burst of activity in collicular visuomotor neurons that lasts at 
least 50ms (Dorris, et al., 2007). Hence distractor effects generated by activity patterns in the intermediate collicular layers do appear to be quite evanescent.

In summary, our findings provide insights into the nature of target selection in saccadic programming. They indicate that the spatial averaging revealed by the presence of distractors in the global effect occurs not in computations related to stimulus location, but in computations of the motor goal, and hence are most likely to occur in neurons with goal-related activity, which are located predominantly in the intermediate layers of the superior colliculus. While our results cannot prove that the superior colliculus is the location of this spatial averaging, our contrasts showing greater global effects for antisaccades than for prosaccades of similar latency are consistent with predictions from a population-coding model of saccade generation in this neural structure. 


\section{References}

Anderson, R. W., Keller, E. L., Gandhi, N. J., \& Das, S. (1998). Two-dimensional saccaderelated population activity in superior colliculus in monkey. $J$ Neurophysiol, 80(2), 798817.

Arai, K., \& Keller, E. L. (2004). A distributed model of the saccadic system: simulations of trajectory variations produced by multiple competing visual stimuli. Conf Proc IEEE Eng Med Biol Soc, 7, 4692-4695.

Arai, K., Keller, E. L., \& Edelman, J. A. (1994). Two-dimensional neural network model of the primate saccadic system. Neural Networks, 7, 1115-1135.

Coeffe, C., \& O'Regan, J. K. (1987). Reducing the influence of non-target stimuli on saccade accuracy: predictability and latency effects. Vision Res, 27(2), 227-240.

Coren, S., \& Hoenig, P. (1972). Effect of non-target stimuli upon length of voluntary saccades. Percept Mot Skills, 34(2), 499-508.

Deubel, H., \& Hauske, G. (1988). The programming of visually guided saccades. In H. Marko, G. Hauske \& A. Struppler (Eds.), Processing structures for perception and action. (pp. 67-74). Weinheim: Verlag Chemie.

Deubel, H., Wolf, W., \& Hauske, G. (1984). The evaluation of the oculomotor error signal. In A. G. Gale \& F. W. Johnson (Eds.), Theoretical and applied aspects of eye movement research (pp. 55-62). Amsterdam: North-Holland.

Dorris, M. C., Olivier, E., \& Munoz, D. P. (2007). Competitive integration of visual and preparatory signals in the superior colliculus during saccadic programming. $J$ Neurosci, 27(19), 5053-5062.

Doyle, M., \& Walker, R. (2001). Curved saccade trajectories: voluntary and reflexive saccades curve away from irrelevant distractors. Exp Brain Res, 139(3), 333-344.

Eggert, T., Sailer, U., Ditterich, J., \& Straube, A. (2002). Differential effect of a distractor on primary saccades and perceptual localization. Vision Res, 42(28), 2969-2984.

Everling, S., Dorris, M. C., Klein, R. M., \& Munoz, D. P. (1999). Role of primate superior colliculus in preparation and execution of anti-saccades and pro-saccades. $J$ Neurosci, 19(7), 2740-2754.

Everling, S., \& Munoz, D. P. (2000). Neuronal correlates for preparatory set associated with prosaccades and anti-saccades in the primate frontal eye field. $J$ Neurosci, 20(1), 387-400.

Findlay, J. M. (1982). Global visual processing for saccadic eye movements. Vision Res, 22(8), 1033-1045.

Findlay, J. M. (1997). Saccade target selection during visual search. Vision Res, 37(5), 617-631.

Findlay, J. M., Brogan, D., \& Wenban-Smith, M. G. (1993). The spatial signal for saccadic eye movements emphasizes visual boundaries. Percept Psychophys, 53(6), 633-641.

Findlay, J. M., \& Gilchrist, I. D. (1997). Spatial scale and saccade programming. Perception, 26(9), 1159-1167.

Glimcher, P. W., \& Sparks, D. L. (1993). Representation of averaging saccades in the superior colliculus of the monkey. Exp Brain Res, 95(3), 429-435.

Godijn, R., \& Theeuwes, J. (2002). Programming of endogenous and exogenous saccades: evidence for a competitive integration model. J Exp Psychol Hum Percept Perform, 28(5), 1039-1054. 
Godijn, R., \& Theeuwes, J. (2004). The relationship between inhibition of return and saccade trajectory deviations. J Exp Psychol Hum Percept Perform, 30(3), 538-554.

Hallett, P. E. (1978). Primary and secondary saccades to goals defined by instructions. Vision Res, 18(10), 1279-1296.

Hallett, P. E., \& Adams, B. D. (1980). The predictability of saccadic latency in a novel voluntary oculomotor task. Vision Res, 20(4), 329-339.

He, P. Y., \& Kowler, E. (1989). The role of location probability in the programming of saccades: implications for "center-of-gravity" tendencies. Vision Res, 29(9), 1165-1181.

Herwig, A., Beisert, M., \& Schneider, W. X. (2010). On the spatial interaction of visual working memory and attention: evidence for a global effect from memory-guided saccades. $J$ Vis, $10(5), 8$.

Lévy-Schoen, A. (1969). Determination et latence de la response oculomotrice a deux stimulus. L'Anee Psychol, 74, 43-66.

Marino, R. A., Rodgers, C. K., Levy, R., \& Munoz, D. P. (2008). The Spatial Relationships of Visuomotor Transformations in the Superior Colliculus Map. J Neurophysiol.

Mays, L. E., \& Sparks, D. L. (1980). Dissociation of visual and saccade-related responses in superior colliculus neurons. J Neurophysiol, 43(1), 207-232.

McSorley, E., Haggard, P., \& Walker, R. (2005). Spatial and temporal aspects of oculomotor inhibition as revealed by saccade trajectories. Vision Res, 45(19), 2492-2499.

Meeter, M., Van der Stigchel, S., \& Theeuwes, J. (2010). A competitive integration model of exogenous and endogenous eye movements. Biol Cybern, 102(4), 271-291.

Mohler, C. W., \& Wurtz, R. H. (1976). Organization of monkey superior colliculus: intermediate layer cells discharging before eye movements. J Neurophysiol, 39(4), 722-744.

Munoz, D., \& Wurtz, R. (1995). Saccade-related activity in monkey superior colliculus I. Characteristics of burst and buildup cells. J Neurophysiol, 73, 2313-2333.

Munoz, D. P., \& Everling, S. (2004). Look away: the anti-saccade task and the voluntary control of eye movement. Nat Rev Neurosci, 5(3), 218-228.

Munoz, D. P., \& Wurtz, R. H. (1995a). Saccade-related activity in monkey superior colliculus. I. Characteristics of burst and buildup cells. J Neurophysiol, 73(6), 2313-2333.

Munoz, D. P., \& Wurtz, R. H. (1995b). Saccade-related activity in monkey superior colliculus. II. Spread of activity during saccades. J Neurophysiol, 73(6), 2334-2348.

Ottes, F., van Gisbergen, J., \& Eggermont, J. (1984). Metrics of saccade responses to visual double stimuli: two different modes. Vision Res, 24, 1169-1179.

Ottes, F. P., Van Gisbergen, J. A., \& Eggermont, J. J. (1985). Latency dependence of colourbased target vs nontarget discrimination by the saccadic system. Vision Res, 25(6), 849862.

Ottes, F. P., Van Gisbergen, J. A., \& Eggermont, J. J. (1986). Visuomotor fields of the superior colliculus: a quantitative model. Vision Res, 26(6), 857-873.

Robinson, D. A. (1972). Eye movements evoked by collicular stimulation in the alert monkey. Vision Res, 12(11), 1795-1808.

Sommer, M. A., \& Wurtz, R. H. (2000). Composition and topographic organization of signals sent from the frontal eye field to the superior colliculus. J Neurophysiol, 83(4), 19792001.

Sparks, D. L., \& Hartwich-Young, R. (1989). The deep layers of the superior colliculus. Rev Oculomot Res, 3, 213-255. 
Trappenberg, T. P., Dorris, M. C., Munoz, D. P., \& Klein, R. M. (2001). A model of saccade initiation based on the competitive integration of exogenous and endogenous signals in the superior colliculus. J Cogn Neurosci, 13(2), 256-271.

Van der Stigchel, S., de Vries, J. P., Bethlehem, R., \& Theeuwes, J. (2011). A global effect of capture saccades. Exp Brain Res, 210(1), 57-65.

Van der Stigchel, S., Meeter, M., \& Theeuwes, J. (2006). Eye movement trajectories and what they tell us. . Neuroscience and Biobehavioral Reviews, 30(5), 666-679.

Van der Stigchel, S., \& Theeuwes, J. (2005). Relation between saccade trajectories and spatial distractor locations. Brain Res Cogn Brain Res, 25(2), 579-582.

Van der Stigchel, S., van Zoest, W., Theeuwes, J., \& Barton, J. J. (2008). The influence of "blind" distractors on eye movement trajectories in visual hemifield defects. J Cogn Neurosci, 20(11), 2025-2036.

Van Gisbergen, J. A., Van Opstal, A. J., \& Tax, A. A. (1987). Collicular ensemble coding of saccades based on vector summation. Neuroscience, 21(2), 541-555.

Van Opstal, A., \& Van Gisbergen, J. A. M. (1990). Role of monkey superior colliculus in saccade averaging. . Exp Brain Res, 79, 143-149.

van Opstal, A. J., \& van Gisbergen, J. A. (1989). Scatter in the metrics of saccades and properties of the collicular motor map. Vision Res, 29(9), 1183-1196.

van Zoest, W., Van der Stigchel, S., \& Barton, J. J. (2008). Distractor effects on saccade trajectories: a comparison of prosaccades, antisaccades, and memory-guided saccades. Exp Brain Res, 186(3), 431-442.

Walker, R., Deubel, H., Schneider, W. X., \& Findlay, J. M. (1997). Effect of remote distractors on saccade programming: evidence for an extended fixation zone. J Neurophysiol, 78(2), 1108-1119.

Walker, R., \& Findlay, J. M. (1996). Saccadic eye movement programming in unilateral neglect. Neuropsychologia, 34(6), 493-508.

Wurtz, R. H., \& Mohler, C. W. (1976). Organization of monkey superior colliculus: enhanced visual response of superficial layer cells. J Neurophysiol, 39(4), 745-765.

Hall, W.C \& Moschovakis, A. (2004). The superior colliculus: new approaches for studying sensorimotor integration. CRC Press, Volume 21 of Methods \& new frontiers in neuroscience series. 


\section{Appendix}

\section{A tectal model of averaging for the Global Effect}

Current explanations of global effects with multiple stimuli and trajectory deviations induced by simultaneous distractors invoke interactions between loci of activity in structures that represent saccadic activity in a spatial map, such as that of the superior colliculus (Van der Stigchel, Meeter, \& Theeuwes, 2006). There is neurophysiological evidence that the superior colliculus is involved in 'weighted averaging' (A. Van Opstal \& Van Gisbergen, 1990), and this weighted averaging plays a prominent role in several models of collicular function (Arai \& Keller, 2004; Arai, Keller, \& Edelman, 1994; Trappenberg, et al., 2001; A. J. van Opstal \& van Gisbergen, 1989). Our knowledge of the spatial map of saccadic coordinates (Robinson, 1972) and the mathematical modeling of saccadic generation (Marino, Rodgers, Levy, \& Munoz, 2008; F. P. Ottes, et al., 1986; Van Gisbergen, Van Opstal, \& Tax, 1987; A. J. van Opstal \& van Gisbergen, 1989) are also more advanced for the superior colliculus than for other ocular motor structures. Therefore, we began by asking whether current models of collicular activity could account for our data.

The first step is to transform our coordinate data into a polar map representing eccentricity and meridional direction. Since the eccentricity of our stimuli and goals is constant at $8^{\circ}$, we simplify our model into a one-dimensional treatment of effects on direction. We transform the directional angles into rostro-caudal tectal coordinates using an equation described in previous studies (Marino, et al., 2008; F. P. Ottes, et al., 1986; Van Gisbergen, et al., 1987):

$$
v=\mathrm{B}_{v} \arctan (\mathrm{R} \sin (\varnothing) /[\mathrm{R} \sin (\varnothing)+\mathrm{A}])
$$

where $v$ is the distance $(\mathrm{mm})$ along the rostro-caudal axis of the superior colliculus, $\mathrm{R}$ is the retinal eccentricity, which is $8^{\circ}$ for our goals, $\varnothing$ is the direction of the goal, and $\mathrm{B}_{v}$ and $\mathrm{A}$ are constants, which have been estimated at $\mathrm{B}_{v}=1.4$ to $1.8 \mathrm{~mm} / \mathrm{rad}, \mathrm{A}=3$ to $5.3^{\circ}$ (F. P. Ottes, et al., 1986; Van Gisbergen, et al., 1987). In this simulation we arbitrarily used Bv $=1.8 \mathrm{~mm} / \mathrm{rad}$ and $\mathrm{A}=3^{\circ}$. A one-dimensional array of rostro-caudal collicular units at this eccentricity can be 
created with units spaced $50 \mu \mathrm{m}$ apart, with maximum directions of $\pm 100^{\circ}$ from the horizontal meridian, for one superior colliculus representing one hemifield (Van Gisbergen, et al., 1987).

Within the two-dimensional collicular spatial map, saccade-related activity is not a point function, but a local 'hill' of activity. Collicular neurons have a tuning function, in that they respond maximally during a saccade to their preferred location, with a decline in their activity as the distance between their preferred location and that of the saccade increases (D. Munoz \& R. Wurtz, 1995). As a result, saccadic activity within the spatial map has a distribution of activity that peaks at the location of the saccade and decays with increasing distance from that peak. This population-coding is often modeled as a bivariate Gaussian function (Trappenberg, et al., 2001; A. J. van Opstal \& van Gisbergen, 1989), a profile that also emerges from neural network models (Arai, et al., 1994) and provides a reasonable fit to neurophysiological data (Anderson, Keller, Gandhi, \& Das, 1998; F. P. Ottes, et al., 1986).

Following these models, one can portray the one-dimensional activity or firing rate ' $\mathrm{C}$ ' of neural units in the map as a Gaussian function of $v$, the direction (mm) in the tectal map. During a saccade to a location on the horizontal meridian,

$$
\mathrm{C}(v)=\mathrm{F}_{\max }\left[\exp \left(-\left(v-\mu_{\mathrm{c}}\right)^{2} / 2 \mathrm{o}_{\mathrm{C}}^{2}\right)\right]=\mathrm{F}_{\max }\left[\exp \left(-(v)^{2} / 2 \text { ó }^{2}\right)\right]
$$

where $\mu_{c}$ is the center of the Gaussian profile, which corresponds to the desired direction of the current saccade, or $0^{\circ}$ for a saccade along the horizontal meridian; ó ${ }_{\mathrm{C}}$ is the tuning width of the Gaussian function, and $\mathrm{F}_{\max }$ is the maximum firing rate, set at 500 spikes/s in some models (Van Gisbergen, et al., 1987). Neurophysiological estimates of ó for prosaccades range from $0.27-0.84 \mathrm{~mm}$ (F. P. Ottes, et al., 1986), with one model setting it at $0.5 \mathrm{~mm}$ (Van Gisbergen, et al., 1987), which is the value we use in this simulation.

A later elaboration of this model, designed in part to give a better account of saccadic averaging from two simultaneous current applications to the colliculus, included functions to represent both lateral intra-collicular inhibitory interactions and non-linear input-output characteristics (A. J. van Opstal \& van Gisbergen, 1989). The expression first characterized C(v) not as firing rate but as current:

$$
\mathrm{C}(v)=\mathrm{E}_{\max }\left[\exp \left(-\left(v-\mu_{\mathrm{c}}\right)^{2} / 2 \text { ó }_{\mathrm{C}}{ }^{2}\right)\right],
$$


where $\mathrm{E}_{\max }$ is $50 \mathrm{mV}$. Inhibition is implemented by a Dirac delta function, which essentially means that all cells except the active one receive a constant level of inhibition. The new equation is:

$$
\mathrm{C}(v)=\mathrm{E}_{\max }\left[\exp \left(-\left(v-\mu_{\mathrm{c}}\right)^{2} / 2 \operatorname{ó}_{\mathrm{C}}^{2}\right)\right]-2 \pi \mathrm{E}_{\max } B \text { ó }_{\mathrm{C}}^{2}
$$

where $B$ is a constant $\left(0.0032 \mathrm{~mm}^{2}\right.$ - for our one-dimensional model, we use $\left.0.056 \mathrm{~mm}\right)$. For the distractor, we model this activity as a second Gaussian function,

$$
\mathrm{D}(v)=\mathrm{E}_{\max }\left[\exp \left(-\left(v-\mu_{\mathrm{D}}\right)^{2} / 2 \mathrm{o}_{\mathrm{D}}^{2}\right)\right]-2 \pi \mathrm{E}_{\max } B \text { ó }_{\mathrm{D}}^{2}
$$

centered at a direction of $\mu_{\mathrm{D}}=20^{\circ}$.

It is assumed that $\mathrm{D}(v)$ and $\mathrm{C}(v)$ make independent contributions to the firing rate of each unit in the one-dimensional collicular array. Thus, when both are present,

$$
\mathrm{W}(v)=\mathrm{D}(v)+\mathrm{C}(v)
$$

The non-linearity input/output characteristic is then imparted by a linear relation between current $\mathrm{W}(v)$ and firing rate $\mathrm{F}(v)$ by:

$$
\mathrm{F}(v)=\beta \mathrm{W}(v)
$$

where $\beta=10$ spikes $/ \mathrm{mV}$, with firing rates less than zero set at 0 and firing rates greater than the $\mathrm{F}_{\max }$ of 500 spikes/s set at 500 .

In a one-dimensional array, where eccentricity is constant, weighted vector-averaging can be reduced to the effects on direction. The movement contribution $\mathrm{M}(i)$ of cell $i$ is related to its location $\mathrm{P}(i)$ and the firing rate given by $\mathrm{W}(i)$, since

$$
\mathrm{M}(i)=a \cdot \mathrm{W}(i) \cdot \mathrm{P}(i),
$$


where $a$ is a fixed scaling constant (spikes/s) ${ }^{-1}$, which thus can be ignored in this simulation. Summation of $\mathrm{M}(i)$ over the entire population of the array $(i=1-n)$ gives the direction of the saccade:

$$
\mathrm{S}=\sum_{\mathrm{i}=1}^{\mathrm{N}}=\mathrm{M}(\mathrm{i})
$$

A key issue is what parameters for $F_{\max }$ (or $E_{\max }$ ) and $o_{c}$, are appropriate for antisaccades. These have not been established, but data show that the firing rate of collicular buildup and burst neurons is about 3 times larger with prosaccades than with antisaccades - see Figure 11 in (Everling, et al., 1999). Models suggest that ó is proportional to $\sqrt{ }(\mathrm{I})$, where I equals the current intensity applied to the deeper layers of the superior colliculus (A. J. van Opstal \& van Gisbergen, 1989), and since firing rate is proportional to I within a range of 0 to $50 \mathrm{mV}$ for I, we propose that $o_{c}$ for antisaccades should equal $\sqrt{ }(1 / 3)$ times the $o_{c}$ for prosaccades, and $F_{\max }$. (or $\mathrm{E}_{\mathrm{max}}$ ) should equal 1/3 of the values for prosaccades (Figure 8).

For $\mathrm{E}_{\max }$ and ó $_{\mathrm{D}}$ for the distractor, we chose values that provided a reasonable fit to the data for prosaccades. That is, we determined what values of $\mathrm{E}_{\max }$ would give rise to the magnitude of prosaccade global effects observed in the different latency bins. Any decrease in $E_{\max }$ was linked to a square-root reduction in ó $\mathrm{D}$. We then examined what these values of $\mathrm{E}_{\max }$

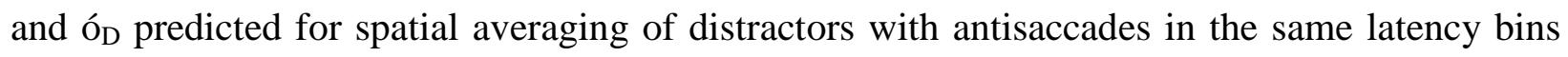
(Figure 7). 

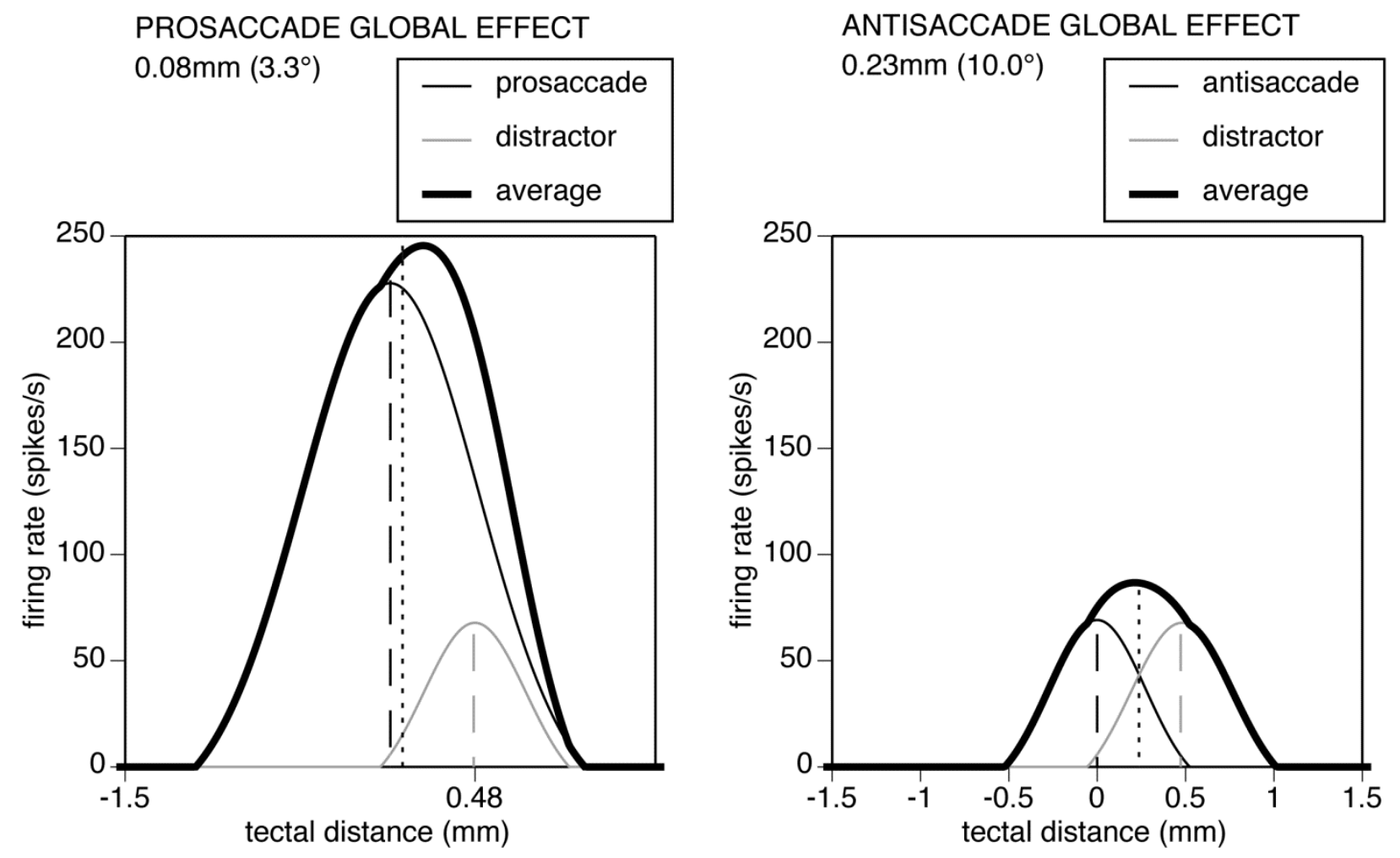

Figure 8. Model of spatial averaging between a saccade on the horizontal meridian and a distractor located $20^{\circ}$ off the horizontal meridian $(0.47 \mathrm{~mm}$ distant in the tectal map). Left graph is for a prosaccade, right graph is for an antisaccade with an activity peak one third that of a prosaccade. In this example, distractor activity (grey line) is modeled as having an activity peak that is also one third that of a prosaccade (black line, left graph). Dashed vertical lines show their peak activity, which is also the center of mass for these isotropic functions. Spatial averaging of saccade and distractor activity (thick black line) results in a shift of the center of activity (dotted vertical lines) away from the horizontal meridian of $0.08 \mathrm{~mm}\left(3.3^{\circ}\right)$ for the prosaccade, and $0.23 \mathrm{~mm}\left(10.0^{\circ}\right)$ for the antisaccade. This approximates reasonably well the empiric findings for the latency bin of $200-240 \mathrm{~ms}$, where the global effect for upper and lower distractors was $2.54^{\circ}$ (s.e. 0.92 ) for prosaccades and $11.36^{\circ}$ (s.e. 3.32) for antisaccades. 